\title{
A rapid and robust assay for detection of S-phase cell cycle progression in plant cells and tissues by using ethynyl deoxyuridine
}

\author{
Edit Kotogány ${ }^{1,2}$, Dénes Dudits², Gábor V Horváth², Ferhan Ayaydin ${ }^{1 *}$
}

\begin{abstract}
Background: Progress in plant cell cycle research is highly dependent on reliable methods for detection of cells replicating DNA. Frequency of S-phase cells (cells in DNA synthesis phase) is a basic parameter in studies on the control of cell division cycle and the developmental events of plant cells. Here we extend the microscopy and flow cytometry applications of the recently developed EdU (5-ethynyl-2'-deoxyuridine)-based S-phase assay to various plant species and tissues. We demonstrate that the presented protocols insure the improved preservation of cell and tissue structure and allow significant reduction in assay duration. In comparison with the frequently used detection of bromodeoxyuridine (BrdU) and tritiated-thymidine incorporation, this new methodology offers several advantages as we discuss here.

Results: Applications of EdU-based S-phase assay in microscopy and flow cytometry are presented by using cultured cells of alfalfa, Arabidopsis, grape, maize, rice and tobacco. We present the advantages of EdU assay as compared to BrdU-based replication assay and demonstrate that EdU assay -which does not require plant cell wall digestion or DNA denaturation steps, offers reduced assay duration and better preservation of cellular, nuclear and chromosomal morphologies. We have also shown that fast and efficient EdU assay can also be an efficient tool for dual parameter flow cytometry analysis and for quantitative assessment of replication in thick root samples of rice.

Conclusions: In plant cell cycle studies, EdU-based S-phase detection offers a superior alternative to the existing Sphase assays. EdU method is reliable, versatile, fast, simple and non-radioactive and it can be readily applied to many different plant systems.
\end{abstract}

\section{Background}

Detection of cell proliferation is a fundamental method for assessing cell health, determining genotoxicity, and evaluating stress responses. The most accurate method utilizes direct measurement of new DNA synthesis. Traditionally, this has been performed by incorporating tritium-labeled thymidine and detection by autoradiography [1]. Because of the involvement of radioactivity, this method has been replaced by incorporation of a thymidine analog such as bromodeoxyuridine (BrdU) into DNA, followed by immunodetection with a specific antibody raised against the thymidine analog [2]. Although being effective, this method requires DNA denaturation or digestion (using

\footnotetext{
* Correspondence: ferhan@brc.hu

${ }^{1}$ Cellular Imaging Laboratory, Biological Research Center, Hungarian

Academy of Sciences, Temesvári krt 62, 6726 Szeged, Hungary
}

hydrochloric acid, heat or DNase) to expose BrdU to the antibody. This step is lengthy, difficult to perform consistently, and can adversely affect the morphology of the sample. Antibody-based detection method of BrdU assay also necessitates cell wall digestion in experiments carried out on plant cells. Therefore protoplasts, partially cell-wall-digested cells and organs or tissue sections are often used for BrdU-based detection of proliferative activity in plants [3].

However, treatment with cell wall digesting enzymes imposes a significant wounding and osmotic stress on plant cells. Moreover, types and concentrations of the enzymes and the osmolarity of the digestion medium should also be specifically optimized for each plant species, organ and cell type under investigation [4]. Partial cell wall digestion or release of protoplasts not only prolong the experimental duration but also cause 
substantial reorganization of cytoskeleton and activation of stress and defense-related genes. To alleviate the stress-related artifacts, it is also possible to first chemically fix the cells and then partially digest cell walls. However, this approach requires highly pure and expensive cell wall digestion enzymes, as crude enzyme preparations contain impurities such as proteases and nucleases that can significantly compromise cellular integrity [5].

EdU (5-ethynyl-2'-deoxyuridine) is a terminal alkynecontaining nucleoside analog of thymidine, and is incorporated into DNA during active DNA synthesis [6]. EdU detection method is based on click chemistry [7]. In a $\mathrm{Cu}(\mathrm{I})$-catalyzed reaction, the alkyne of EdU reacts with an azide containing fluorochrome, forming a stable covalent bond. EdU-based assay has been successfully used in detection of proliferation in avian cochlea [8], in chick embryos [9] in breast cancer cells [10] and in human fibroblasts [11]. Twenty-four hours long EdU incubation duration has been recently used in Arabidopsis root tips to identify dysfunction of the quiescent center [12], but the possibility of very short EdU pulse labeling to determine S-phase indices, the suitability of this novel detection method in various plant species, comparison of EdU assay to BrdU assay and plant specific parameters and fields of application such as plant flow cytometry have not been explored in detail.

Here we show the advantages in microscopy and flow cytometry applications of this novel S-phase detection assay using various cultured plant cells and root meristems.

\section{Results and Discussion}

\section{EdU-based assay versus immunodetection of BrdU}

To compare EdU assay with BrdU immunodetection, Arabidopsis thaliana sp. Columbia suspension culture cells were incubated with $10 \mu \mathrm{M}$ EdU for $2 \mathrm{hrs}$ and fixed with detergent-containing formaldehyde. Since EdU detection does not rely on antibodies and the molecular size of the chemicals used during detection is small compared to the size of the antibody molecules, cells were directly incubated with the detection cocktail without performing cell wall digestion. The detection cocktail is composed of an azide-conjugated fluorochrome (such as Alexa Fluor 488) and copper (I) as the catalyzer of the click reaction [13]. Stained nuclei with high signal-to-noise ratio were easily detectable under the microscope (Figure 1A). Omission of cell wall digestion and DNA denaturation/digestion steps resulted in better preservation of cellular structures judged by DAPI (4',6-diamidino-2-phenylindole) staining of the nuclei and by differential interference contrast (DIC) microscopy (Figure 1A). As comparison, $10 \mu \mathrm{M}$ (2 hrs) BrdU-labeled and similarly fixed Arabidopsis cells were processed for immunodetection of BrdU. Following fixation washes, cell walls were partially digested with an enzyme cocktail composed of high purity cellulase and pectinase. Cells were settled on poly-L-lysine coated slides and plasma membranes were permeabilized with Triton X-100 followed by DNase I digestion. Anti-BrdU antibody incubation was followed by fluorochrome-conjugated secondary antibody incubation. Similar to EdUlabeled nuclei, BrdU-labeled nuclei were also detectable albeit with high background noise (Figure 1B). Cell wall digestion and DNase treatment often resulted in partial loss of globular cell shape and barely detectable nuclei with a melted look (Figure 1B arrows). On the other hand, EdU assay resulted in morphologically well-preserved cells with clearly identifiable nuclei brightly stained with the DNA dye DAPI (Figure 1A). Clear determination of the total number of nuclei following EdU assay was particularly advantageous for correct assessment of S-phase index. Moreover, total experimental duration of EdU assay was calculated to be 6 hours shorter than that of BrdU immunolabeling procedure (Table 1). On the other hand, one unique advantage of immunodetection-based assay is that, availability of specific antibodies against halogenated thymidine analogs such as, chlorodeoxyuridine, iododeoxyuridine as well as bromodeoxyuridine allows multiplexing experiments to analyze replication initiation and temporal dissection of S-phase [14]. Sharpless and coworkers reported the ruthenium-catalyzed cycloaddition of azides to alkynes [15]. While the $\mathrm{Cu}(\mathrm{I})$-catalyzed EdUbased click reaction is limited to terminal alkynes, the $\mathrm{Ru}(\mathrm{II})$-catalyzed reaction is active with internal alkynes, as well. Therefore, similar multiplexing experiments may also be possible with the future development of EdUanalogous chemicals that have internal alkyne groups.

EdU assay is a versatile method for both monocot and dicot plant species

The thickness as well as the composition and organization of plant cell walls can vary significantly between species and also differ between cell types and developmental stages [16]. Therefore, we have tested EdU assay on various monocot and dicot plant suspension cultures. Two days after subculturing, alfalfa, Arabidopsis, grape, maize, rice and tobacco cells were treated for 2 hours with $10 \mu \mathrm{M}$ EdU, fixed and incubated with EdU detection cocktail. Nuclei were counterstained with DAPI and cells were analyzed by confocal laser scanning microscope (Figure 2). EdU-labeled nuclei were clearly detected in all cultured cells tested. While cells that were just entered into S-phase after the addition of EdU showed complete labeling of the nuclei, those cells which were entered into replication phase near to the end of the 2 hours labeling period showed spotty labeling (Figure 2A, B, D, F, arrows in green panel). These 
Table 1 Comparison of experimental steps and durations for EdU-assay and BrdU-based immunolocalization

\begin{tabular}{ccc}
\hline & EdU assay & BrdU immunolocalization \\
\hline EdU or BrdU labeling & $2 \mathrm{~h}^{*}$ & $2 \mathrm{~h}^{*}$ \\
\hline Fixation and washes & $15 \mathrm{~min}+15 \mathrm{~min}$ min $+15 \mathrm{~min}$ \\
\hline Cell wall digestion and washes & - & $30 \mathrm{~min}+15 \mathrm{~min}$ \\
\hline Plasma membrane permeabilization and washes & - & $30 \mathrm{~min}+15 \mathrm{~min}$ \\
\hline DNase I digestion and washes & - & $30 \mathrm{~min}+15 \mathrm{~min}$ \\
\hline Washing with DAPI and sample mounting & $30 \mathrm{~min}$ & $3 \mathrm{~h}+25 \mathrm{~min}+1 \mathrm{~h}$ \\
\hline Total experimental duration & $20 \mathrm{~min}$ & $20 \mathrm{~min}$ \\
\hline
\end{tabular}

* Depending on the proliferation activity of a given cell type, shorter incubations may be sufficient or longer incubations may be necessary.

patchy-labeled early S-phase nuclei were well preserved due to absence of harsh treatments during the detection protocol. Mild reaction conditions were also favorable from the point of superior preservation of chromosomal morphology in mitotic cells (Figure 2A, D, arrowheads and Figure 3 inset). This was particularly helpful to assess proliferation both by S-phase index and by mitotic index. Dual parameter analysis of the cell cycle for the tested cultures indicated that maize culture has the highest number of S-phase cells followed by Arabidopsis and alfalfa cultures after two days of subculturing
(Figure 3). Relatively low DNA synthetic activity was detected for tobacco (SR1) and rice (Unggi 9) cultures after 2 hrs EdU incubation. Lowest frequency of S-phase cells was detected in grape culture, which can be explained by the recent initiation of this cell line (Figure 3 ). The differences in proliferation activity among the cultures may be explained by differences in cell cycle durations and/or differential adaptation to liquid culture and hormone conditions. To determine the relationship between EdU concentration and labeling duration, we have treated Arabidopsis cultures with various EdU

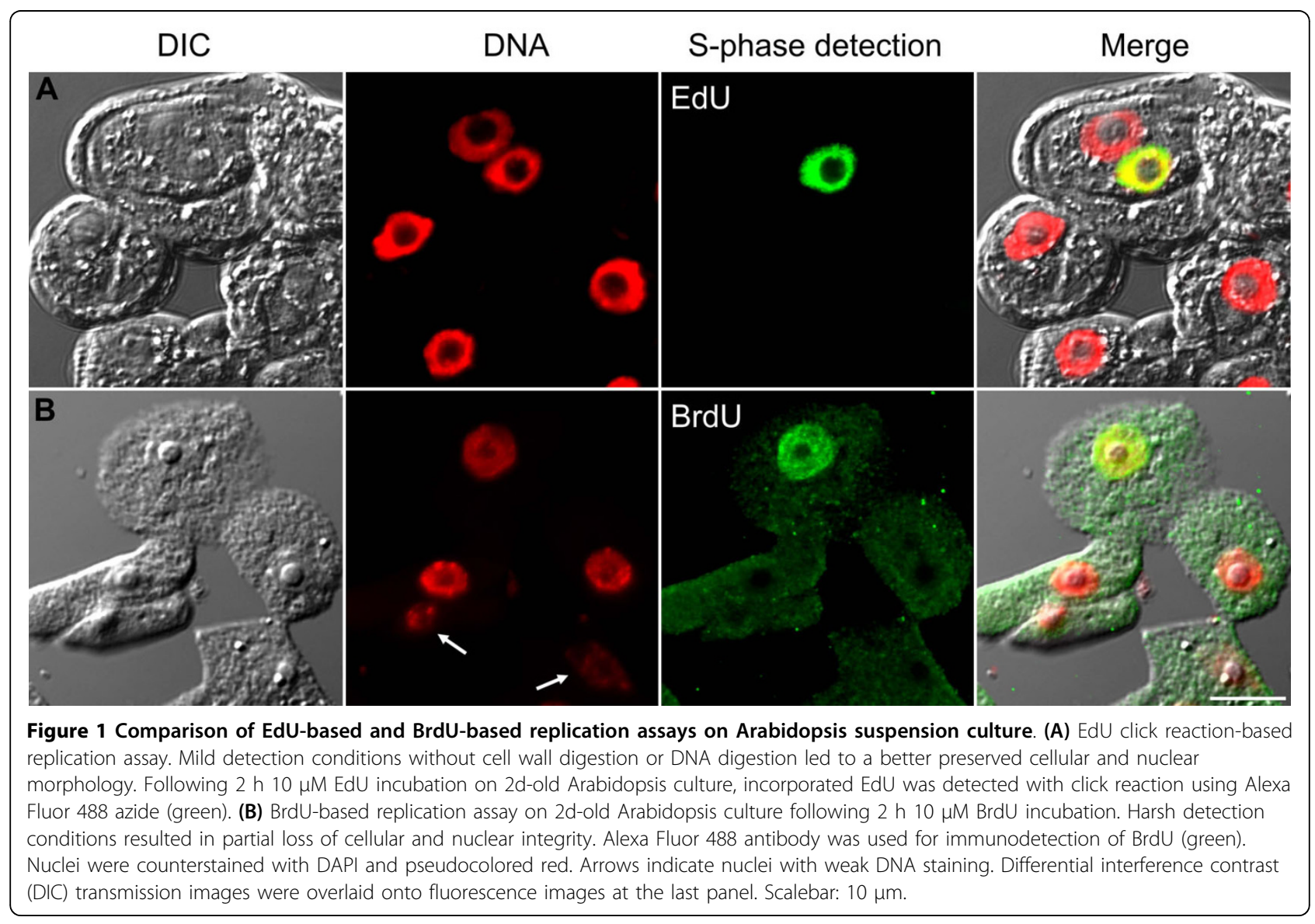


concentrations for different labeling durations (Figure 4). Incubation durations as short as 15 minutes were possible using $10 \mu \mathrm{M}$ or $50 \mu \mathrm{M}$ EdU concentrations for the fast growing Arabidopsis culture after $36 \mathrm{hrs}$ of subculturing. For $1 \mu \mathrm{M}$ EdU concentration, at least $30 \mathrm{~min}-$ utes incubation was necessary to obtain easily detectable signal intensity. Using less than $1 \mu \mathrm{M}$ EdU concentration was not sufficient for S-phase index determination due to extremely low fluorescence intensities of the labeled nuclei (Figure 4, $0.1 \mu \mathrm{M}$ and $0.01 \mu \mathrm{M}$ ). Based on the values of Figure 3 ( $10 \mu \mathrm{M}$ EdU, 2 hrs labeling), we predict that for slow growing cultured cells having 2-3 times less EdU-labeling index than that of Arabidopsis cells, 30 to 60 minutes-long incubation with at least 10 $\mu \mathrm{M}$ EdU concentration may be needed as minimum labeling duration. On the other hand, longer incubations can also be used to assess the frequency of cells, which entered and exited S-phase during the labeling period. Due to its cumulative effect, this technique can be especially useful to determine fine differences in S-phase regulation as well as to assess the length of G2 phase by determining minimum incubation duration needed for the first appearance of EdU-labeled mitotic chromosomes. Nevertheless, simple and versatile EdU assay can be used to rapidly screen the proliferation status of several in vitro plant cultures as well as to assess proliferation response after various treatments or genetic modifications.

\section{Using EdU assay in flow cytometry of plant nuclei}

Cell-cycle-phase distribution including DNA synthesis activity of a given in vitro culture or in vivo organ can effectively be determined by flow cytometry after isolation of nuclei. In animal cell cultures, high quality and intact nuclei preparations can be relatively easily obtained in hypotonic buffers with detergents. In plants, however, cell walls pose a significant barrier for nuclei isolation. To alleviate this problem, cell walls can be removed by protoplast isolation followed by mechanical disruption in hypotonic buffers with detergents. However, removal of cell walls can require hours during which period cells cease entering into mitosis or DNA replication phase due to severe wounding stress and osmotic shock. Therefore, this method is not suitable for determining kinetic parameters of cell division cycle. As an alternative, Galbraith et al. [17] used a practical approach in which the suspensionsof intact nuclei are prepared by chopping a small amount offresh tissue in a suitable isolation buffer. This method affords high quality DNA histograms and provides immediate release of nuclei from cells or tissues and is currently widely used for flow cytometry of unfixed nuclear samples of plant cells $[18,19]$. Formaldehyde fixation of samples offers convenience in experiments involving multiple samples and timepoints such as cell cycle synchronization.
However, fixed nuclear preparations often display wider G1 and G2 peaks in flow cytometry histograms making uniparametric S-phase estimations difficult. As an alternative to single parameter flow cytometry analysis, BrdU immunodetection combined with DNA staining can be used as biparametric (bivariate or dual-parameter) analysis of fixed nuclei for reliable determination of various cell cycle phases and cell cycle dynamics without using estimation algorithms [20]. Similar to microscopy-based immunodetection of BrdU, detection of BrdU in flow cytometry also requires DNA denaturation before the use of antibodies. Since excessive DNA denaturation adversely affects the quality of the nuclei, the concentration, duration and temperature of the digestion or denaturation process need to be meticulously optimized $[20,21]$. Moreover, treatment with antibodies prolongs the overall experimental duration and adds an extra step of complexity. Due to its simplicity, EdU-based method can be a superior alternative to BrdU-based flow cytometry especially in plant cell studies. EdU-based biparametric flow cytometry has recently been reported in breast cancer cells [10] and its efficiency has been demonstrated as compared to BrdU-based flow cytometry assay in human leukemia cells [13], however, its use in plant cell flow cytometry, to our knowledge, has not been reported, yet. We have used a monocot (rice) and two dicot (alfalfa, Arabidopsis) suspension cultures for biparametric flow cytometry analysis (Figure 5). After 10 $\mu \mathrm{M} \mathrm{EdU} \mathrm{(or} \mathrm{0.1 \%} \mathrm{DMSO)} \mathrm{treatment,} \mathrm{cultures} \mathrm{were}$ directly chopped with a razor blade in nuclei isolation buffer to release nuclei. Formaldehyde fixed (Figure 5A$D$ and 5G) or unfixed (Figure 5E, F) samples were incubated in EdU detection cocktail followed by DNA counterstaining with DAPI (for microscopy) or with propidium iodide (for flow cytometry). Using an aliquot of these samples, EdU-labeled nuclei were checked for quality of labeling as well as integrity of nuclei by DAPI staining. (Figure 5 inset photos). Remaining nuclei preparations were analyzed by uniparametric (DNA content by propidium iodide, Figure 5 upper panels) and by biparametric flow cytometry (Alexa Fluor 488-EdU intensity versus DNA content, Figure 5 lower panels). After 3 days of subculturing, rice cells treated with EdU for eight hours displayed $12.3 \%$ EdU labeled nuclei as a clearly separated cluster in bivariate dot-plot (Figure $5 \mathrm{~B})$. Based on their DNA content, the nuclei of this cluster represent the cells that were in early, mid and late S-phase in addition to the cells that already entered into G2 phase during the labeling duration. For faster growing alfalfa cells (3d-old) which were incubated for 4 hours with EdU, this cluster displayed nearly 17\% EdUlabeled nuclei (Figure 5D). To determine nuclear autofluorescence and non-specific binding of Alexa Fluor 488 azide, control cultures were incubated with $0.1 \%$ 


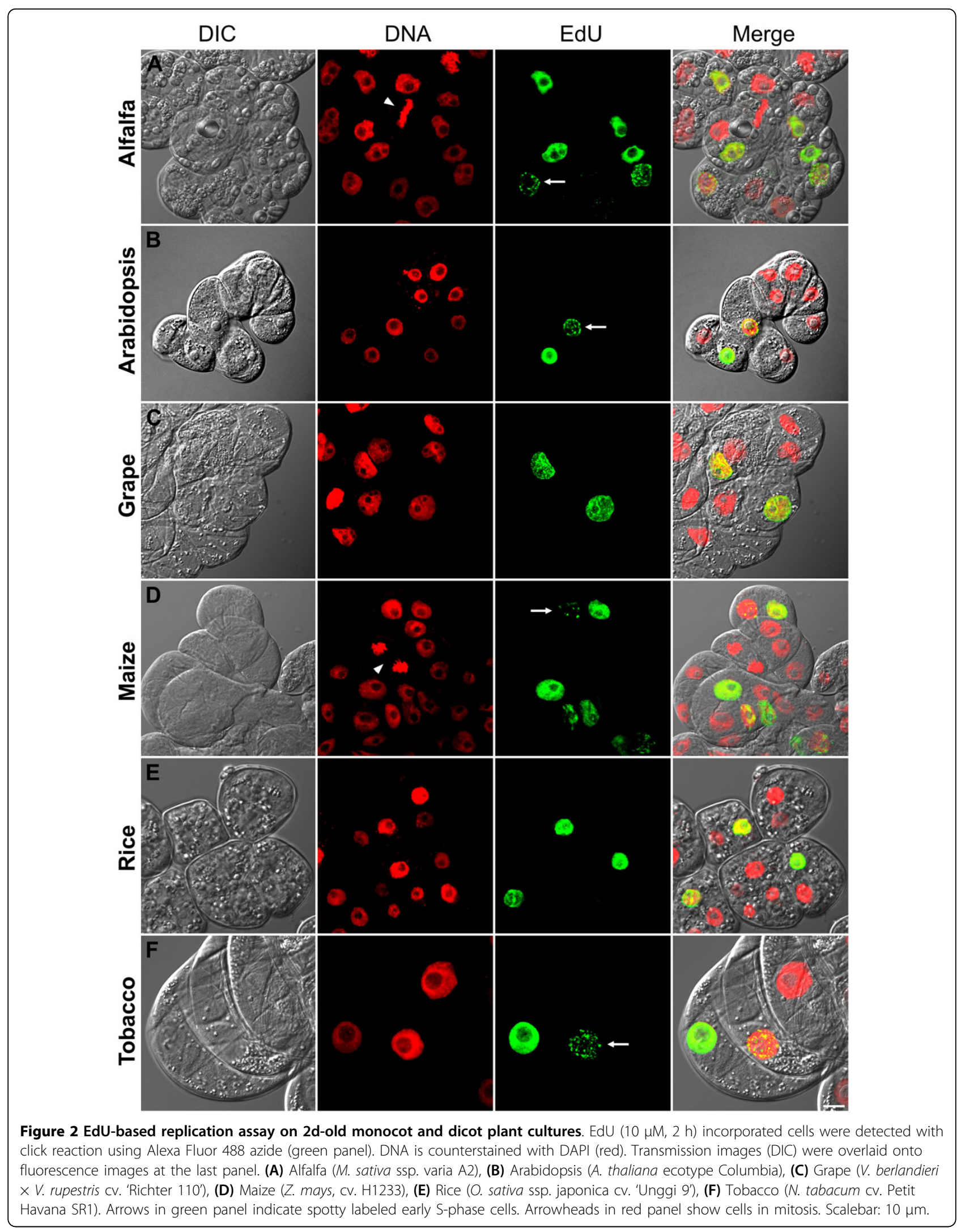




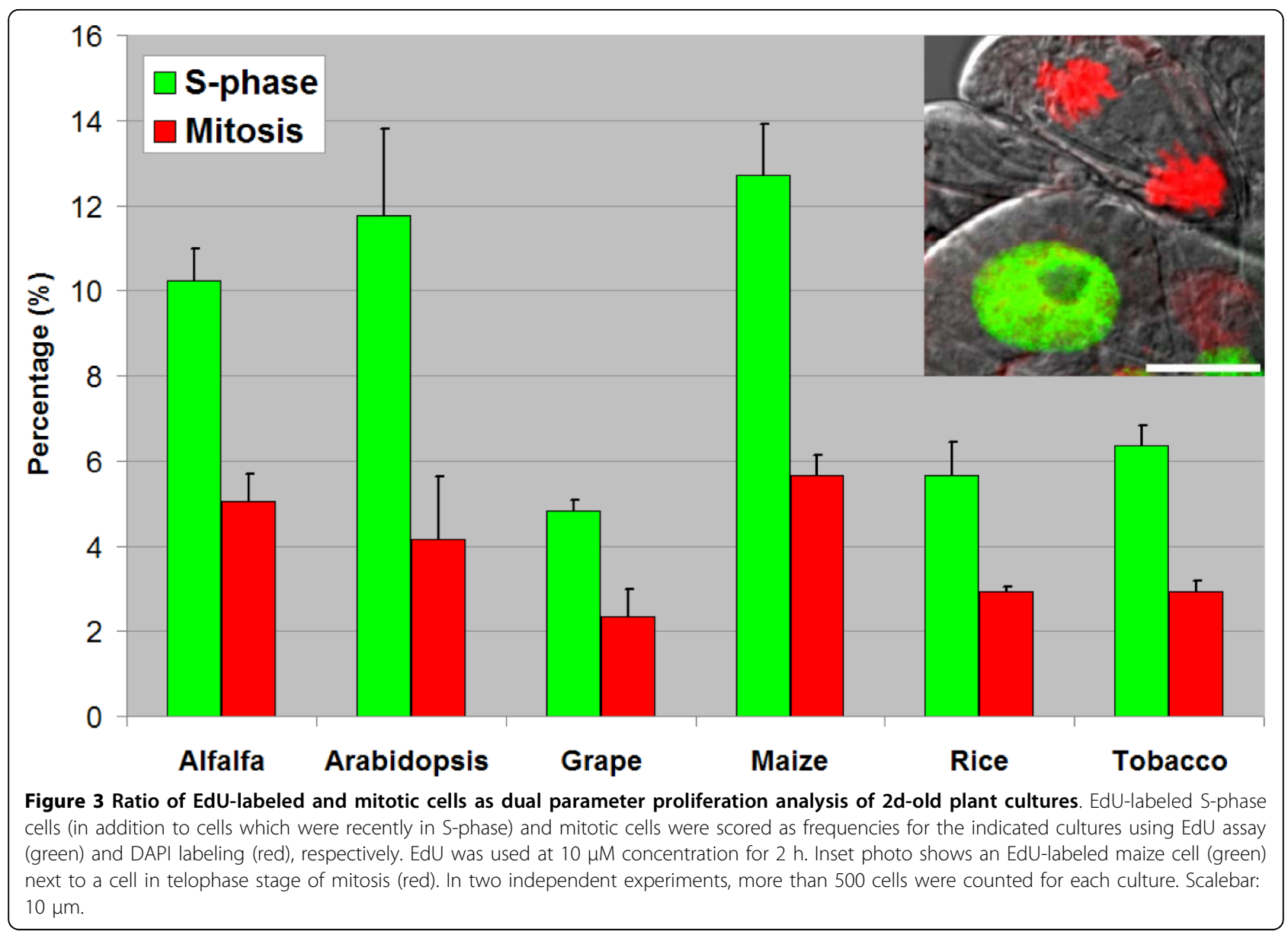

DMSO (solvent of EdU) and similarly processed as in EdU-treated samples. Less than $0.3 \%$ of the total nuclei were detected above the "EdU threshold" value (see Methods) indicating the specificity of the assay (Figure 5A, C, E and DMSO in 5G). Unlike antibody-based BrdU assay, click reaction should not be sensitive to cellular proteases released during chopping procedure; therefore, we have tested the suitability of EdU assay (10 $\mu \mathrm{M}$ EdU, $4 \mathrm{~h}$ ) on unfixed nuclei of $4 \mathrm{~d}$-old rice cultures. Fresh nuclear preparations should be kept cold to prevent degradation of samples. While EdU click reaction was not efficient on ice-cold temperatures, after $30 \mathrm{~min}$ utes incubation at room temperature, we could successfully detect EdU incorporated nuclei both by flow cytometry and by microscopy (Figure 5F). Unfixed nuclei displayed significantly better monoparametric histograms (Figure 5E, F, upper panels) with narrower G1 and G2 peaks. On the other hand, on several independent experiments, we have observed noticeably higher signal intensities of EdU-labeled fixed nuclei as compared to unfixed nuclei. Nevertheless, from chopping of cells to flow cytometry analysis, the total duration of biparametric EdU assay was considerably shorter than
BrdU assay using either fixed (1.5 hrs) or unfixed (45 mins) nuclei (see Methods). To assess the minimum feasible EdU incubation duration, we have tested 15 and 30 minutes of EdU incubations $(10 \mu \mathrm{M})$ on 3-days-old Arabidopsis cultures. Incubation durations as short as 15 and 30 minutes were enough to obtain labeling indices higher than DMSO treated control nuclei (Figure 5G, Total EdU+).

Based on these experiments, the advantages of EdU assay for use in plant flow cytometry were readily evident. Clearly defined EdU-labeled population on a logarithmic scale in biparametric plots allowed us to precisely determine the fraction of cells labeled with EdU, hence giving information on the dynamics of Sphase entry and progression of cells into G2 phase of the cell cycle. Especially with short EdU pulse durations, dual parameter analysis with EdU is very informative and advantageous as subpopulations of S-phase (early, mid and late S-phase) can be better assessed. As an example, Figure $5 \mathrm{~B}$ displays a simple quadrant analysis. Q1 and Q2 sectors indicate the population of $2 \mathrm{C}$ and $4 \mathrm{C}$ nuclei that never entered into replication phase during EdU labeling period. Q3 fraction represents nuclei 


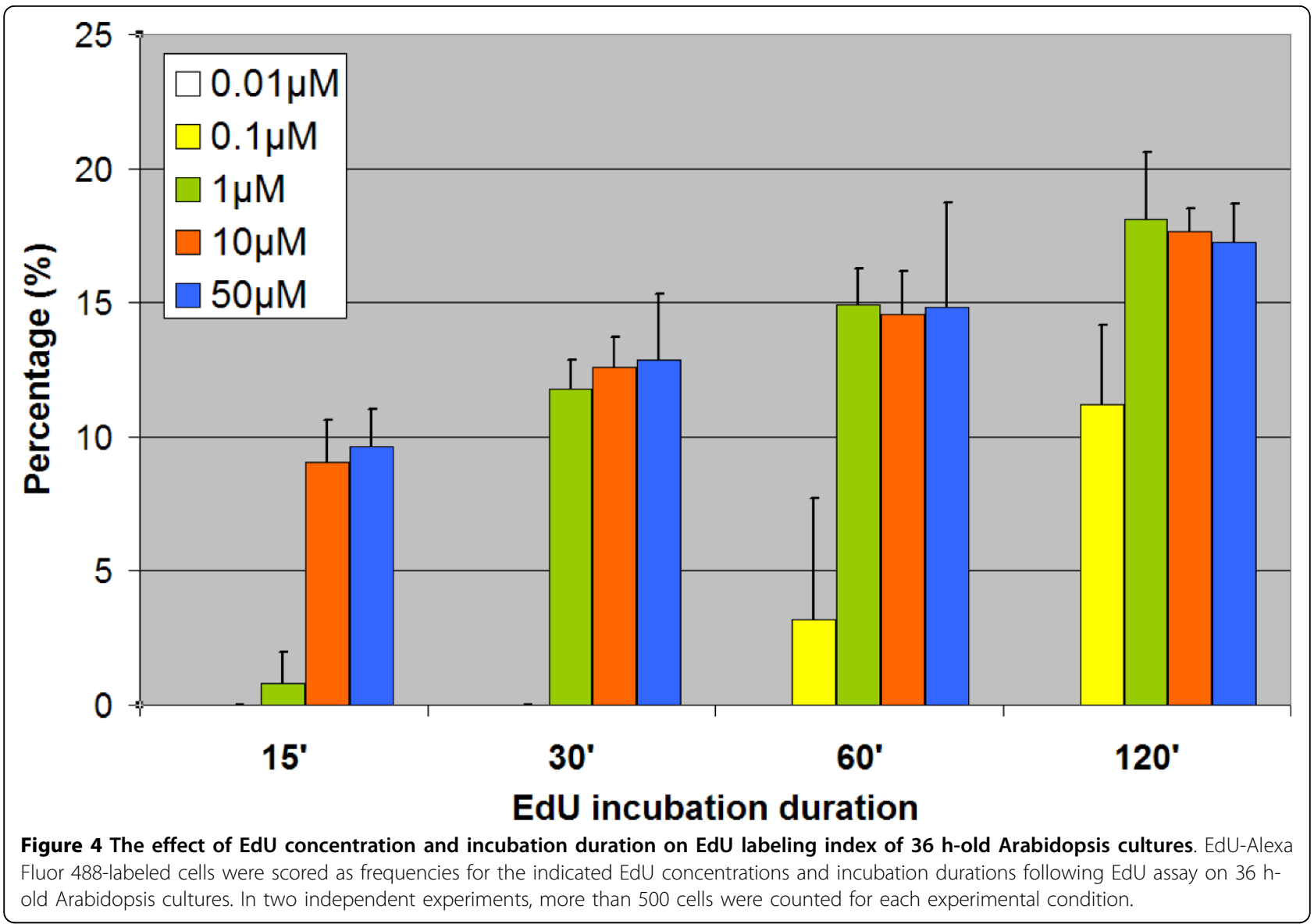

entered into S-phase relatively recently. Q4 fraction indicates nuclei entered into S-phase earlier than that of Q3 fraction during the EdU labeling period. This fraction contains nuclei in mid to late S-phase in addition to EdU-labeled nuclei that already entered into G2 phase with 4C DNA content.

In summary, the small size of molecules participating in the EdU labeling and detection allows for fast and efficient detection of the incorporated EdU without using harsh conditions, which may adversely affect the quality of data especially in plant flow cytometry. Simple and efficient EdU-based biparametric flow cytometry can therefore be readily incorporated into studies involving both monocot and dicot plant species and can be a very effective tool to assess culture health and proliferation status or in experiments based on plant cell cycle synchronization, stress treatments and genetic modifications. EdU analysis of S-phase cells in rice root meristems Immunolocalization of BrdU on thick plant tissues with dense cell layers is especially difficult due to cell wall and plasma membrane penetration problems of large $(150 \mathrm{kDa})$ antibody molecules. To address these limitations, mechanical sectioning of plant material has been adopted [22]. However, sectioning protocol is tedious, time-consuming and requires expertise and specialized equipment. Moreover, reconstruction of the original three-dimensional tissue conformation from the individual sections is complicated. Click-reaction based EdU proliferation assay uses a small fluorochrome molecule $(0.6 \mathrm{kDa})$ for detection [13]. Following successful application of EdU proliferation assay in various cultured cells with intact cell walls (Figure 2), we have tested EdU labeling on intact roots of germinating rice seedlings which have considerably thicker roots than, for example, Arabidopsis seedlings. Root tips of germinating rice were incubated for 2,4 and 6 hours with $20 \mu \mathrm{M}$ EdU then fixed with formaldehyde in a detergent-containing buffer and finally washed and incubated with EdU-detection reagent. EdU-labeled meristematic regions of roots were easily detectable with a fluorescence stereo microscope (Figure 6A). For detailed analysis of the meristem region and quantitation of the signal intensity, we have used confocal laser scanning microscope (Figure 6B). Single optical sections of $6 \mu \mathrm{m}$ (optical depth) on the median plane of rice root tips were captured and average green fluorescence intensity of the $500 \mu \mathrm{m}$-long conical meristem area was measured and plotted (Figure 6D). Root contours, quiescent center and 


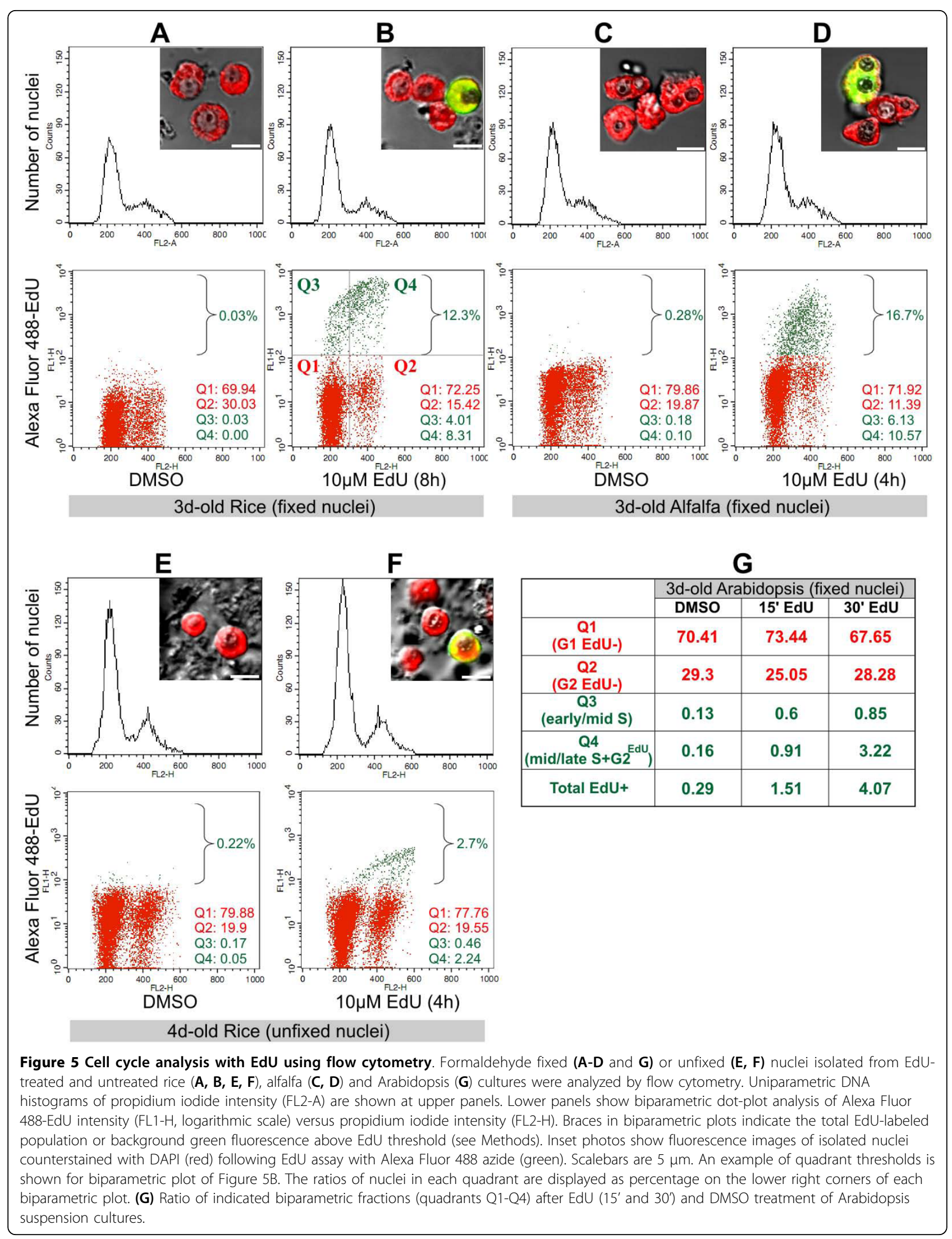


columella cells were identified by lignin autofluorescence of the cell walls [23]. No proliferation activity was detected in the quiescent center or in columella cells even after 6 hours of EdU incubation (Figure 6B). In the meristem region, however, EdU incubation time-dependent intensity increase was easily visible and quantifiable. Since EdU-detection protocol does not require acid or heat denaturation or digestion of DNA, chromosome morphology of the meristem region was also perfectly preserved. After DNA counterstaining, scoring of mitotic and EdU-labeling indices was also possible on individual optical sections with clearly defined mitotic phases (Figure 6C), however global measurement of average EdU incorporation in the meristem zone proved to be a faster and more convenient way for determination of proliferation activity in thick root tips of rice. Unlike elongation and differentiation zones of roots, the root meristematic zones display high proliferation activities with short cell cycle times. EdU labeling durations of 4 and 6 hours resulted in many EdU-labeled mitotic figures in root tips of rice. Inset photo of Figure 6B shows EdU-labeled anaphase chromosomes after 4 hours of EdU incubation. Presence of such post-metaphase figures also suggests that the incorporation of EdU into DNA does not cause chromosome segregation abnormalities during mitosis. Since EdU-labeled mitotic figures were detected very rarely after $2 \mathrm{~h}$ EdU incubation, we predict that the average $\mathrm{G} 2$ phase duration in rice root tip meristems (O. sativa L. ssp. japonica 'Nipponbare') should be between 2 and 4 hours. To determine the relationship between EdU concentration and labeling duration, rice root tips were treated with $0,2,20$ and $100 \mu \mathrm{M}$ EdU for 15, 30 and 60 minutes (Figure 7). Similar to the experiments with Arabidopsis suspension cultures (Figure 4), higher EdU concentrations allowed shorter EdU pulse durations. Comparable EdU labeling intensities at the root meristem regions were obtained with 15, 30 and 60 minutes of EdU incubations using 100,20 and $2 \mu \mathrm{M} \mathrm{EdU}$, respectively (Figure 7). Successful application of EdU assay on relatively thick rice root tip meristems suggests that the protocol can be applied to other plant organs such as leaves, seeds, shoot meristems, stems or flowers using the delivery methods already optimized for BrdU assays such as floating of seedlings [24], imbibition of seeds [25], liquid culturing of excised inflorescences [26] and direct submerging of intact leaves [27]. Practical and quantitative nature of root proliferation assay will find its application in various fields of plant science research such as developmental biology and stress physiology. Moreover, use of low magnification fluorescence stereo microscopy allows rapid screening of various experimental conditions therefore can be particularly suitable for high throughput screening studies.

\section{Conclusions}

The EdU-based S-phase assay presented here affords a simple and rapid yet robust complement to previously validated methods of proliferation analysis in plant cells. Unlike antibody-based BrdU assay, EdU-based S-phase assay does not require cell wall digestion or DNA denaturation. This is not only advantageous from the point of morphological preservation of the samples, but also saves considerable amount of experimental time. EdU labeling and detection protocol can easily be adapted to various plant species regardless of cell wall thickness, composition or structure. The assay will be particularly useful in plant flow cytometry analyses due to superior preservation of isolated nuclei during the detection protocol. Apart from its application in cultured cells and isolated nuclei, the method is also well suited for quantitative proliferation analysis on thick tissues such as roots. Taking these data into account, we conclude that practical, versatile and quantitative nature of this robust S-phase assay will find its application in various fields of plant science research and will certainly be the new gold standard replacing BrdU-based immunolocalization assays.

\section{Methods}

\section{Plant growth conditions}

Plant suspension cultures were subcultured weekly. Culture medium and hormone concentrations are shown in Table 2. Rice (O. sativa ssp. japonica 'Nipponbare') seeds were surface sterilized for 5 min with $5 \%$ sodium hypochlorite solution (undiluted commercial bleach) then for 30 seconds with $70 \%$ ethanol solution. Seeds were washed $3 \times 10 \mathrm{~min}$ with sterile distilled water and soaked in distilled water until germination started (24$36 \mathrm{~h}$ ). Germinating seeds were transferred onto Petri dishes containing $1 \%$ agar in half strength MS medium (1/2 MS). Petri dishes were kept near vertically to promote unidirectional growth of root tips.

\section{Immunolocalization of BrdU}

BrdU (5-bromo-2'-deoxyuridine, Sigma catalog no: B5002) stock solution was prepared as $30 \mathrm{mM}$ aliquot in DMSO and kept in freezer. Two-days-old suspension culture of Arabidopsis was incubated for $2 \mathrm{hrs}$ with $10 \mu \mathrm{M}$ BrdU in its own culture medium. Cells were then fixed $15 \mathrm{~min}$ in $4 \%(\mathrm{w} / \mathrm{v})$ formaldehyde solution in phosphate buffered saline (PBS) with $0.1 \%$ Triton X-100. Eight percent $(2 \times$ concentrated) formaldehyde stock solution was prepared as following: Paraformaldehyde powder (Fluka cat. no: 76240 ) was dissolved in water by heating to about $60^{\circ} \mathrm{C}$ inside a fume hood and a drop of concentrated $\mathrm{KOH}$ was added as heating and alkaline $\mathrm{pH}$ depolymerizes paraformaldeyde. After cooling to room temperature, $\mathrm{pH}$ was set to neutral $\mathrm{pH}$ with dilute $\mathrm{H}_{2} \mathrm{SO}_{4}$ [5]. Aliquots of this stock fixer can be frozen for a few months. This $2 \times$ 


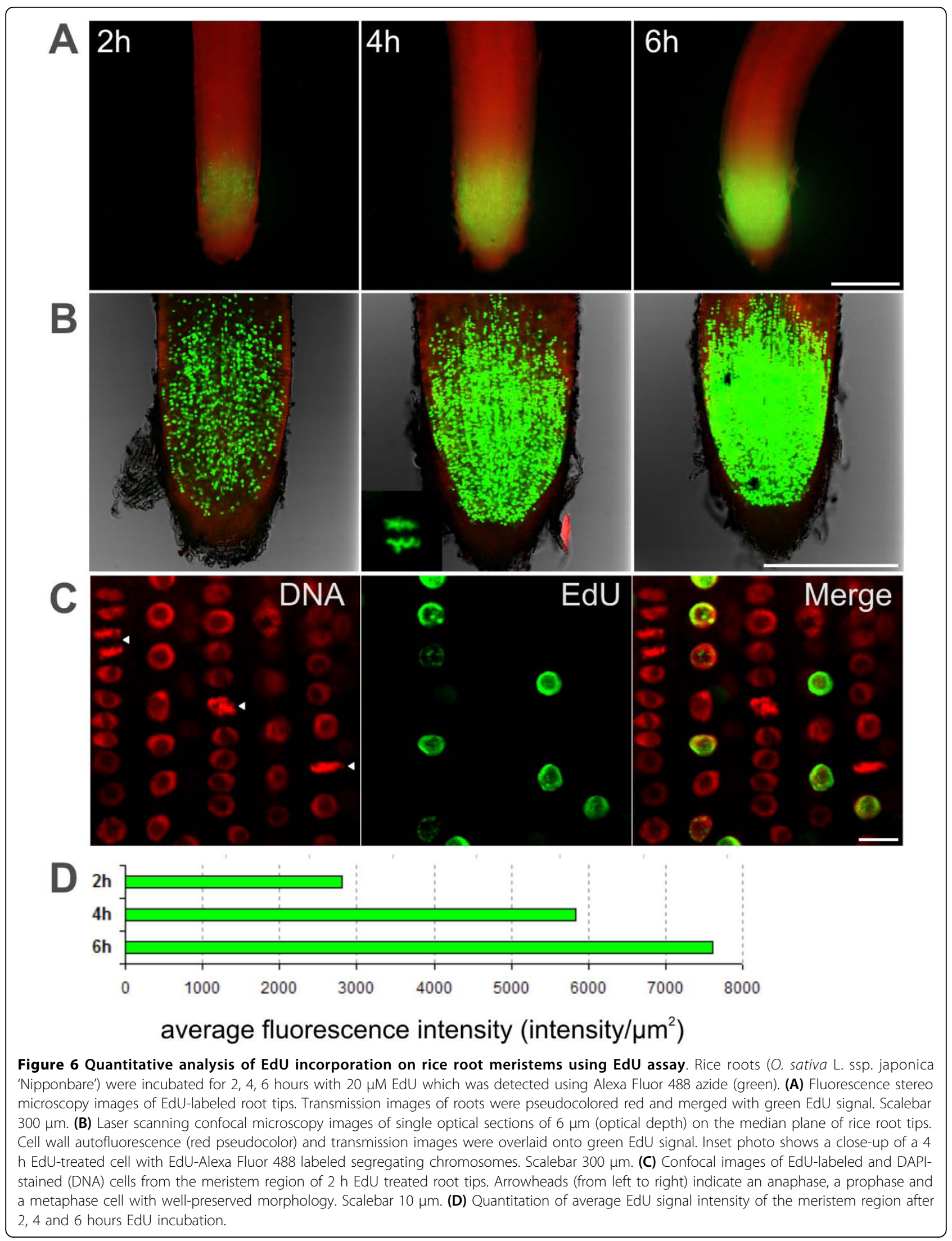




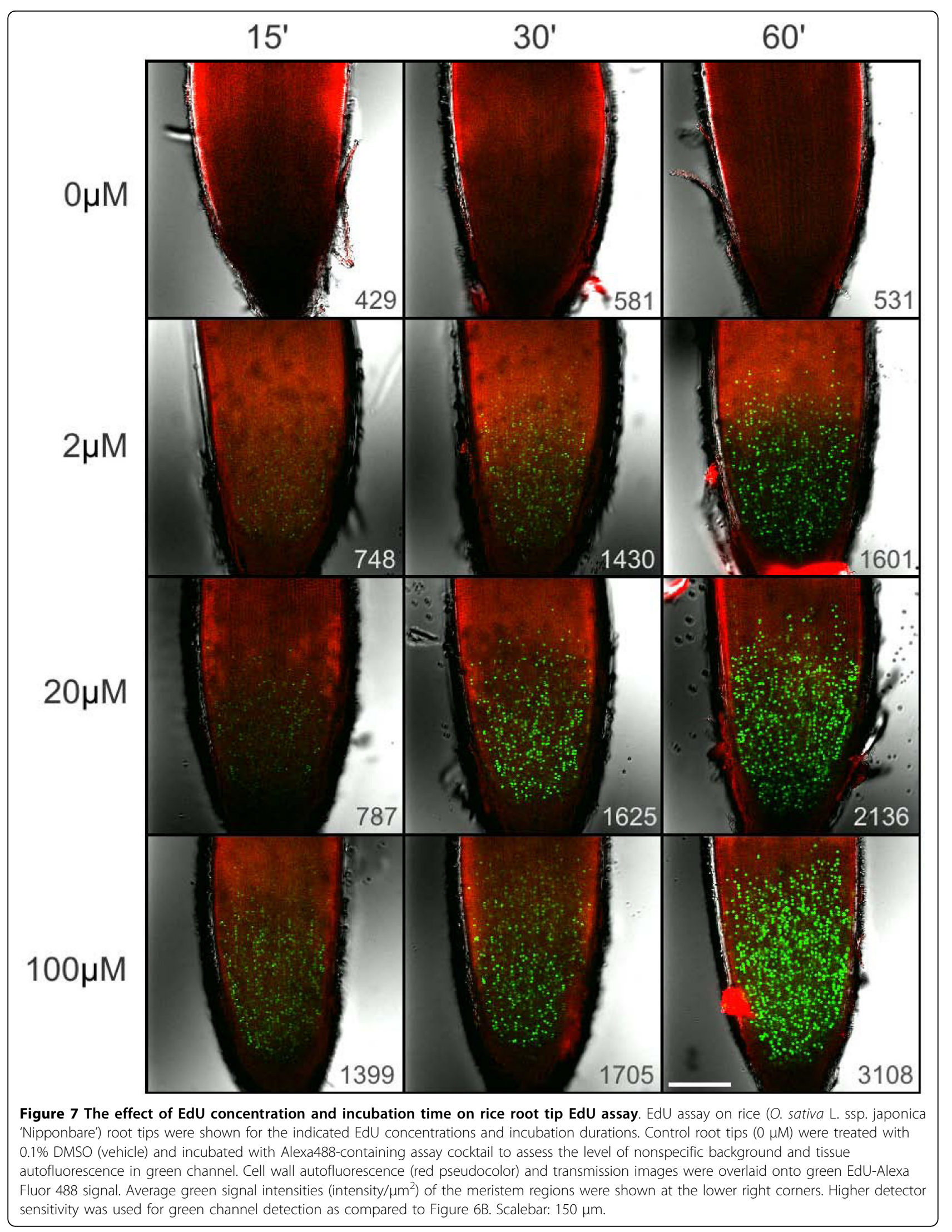


Table 2 Hormone concentrations and media used for plant cultures.

\begin{tabular}{ccccc}
\hline & Growth Medium & 2,4-D (mg/L) & NAA (mg/L) & Kinetin (mg/L) \\
\hline Alfalfa (M. sativa ssp. Varia A2) & MS & 1 & - & 0.2 \\
\hline Arabidopsis (A. thaliana ecotype Columbia) & MS & - & 0.5 & 0.05 \\
\hline Grape (V. berlandieri $\times$ V. rupestris cv. 'Richter 110') & MS & - & 1 & - \\
\hline Maize $(Z$. mays, cv. H1233) & N6 M (LP40) & 0.5 & - & - \\
\hline Rice (O. sativa ssp. japonica cv. 'Unggi 9' & G1 & 1 & 1 & - \\
\hline Tobacco (N. tabacum cv. Petit Havana SR1) & MS & - & 0.2 \\
\hline
\end{tabular}

2,4-D: 2,4-Dichlorophenoxyacetic acid. NAA: 1-Naphthaleneacetic acid. MS: Murashige and Skoog medium [29]. N6 M (LP40): Maize culture medium [30] with 40 $\mathrm{mg} / \mathrm{L}$ L-proline. G1: Rice culture medium [31,32]

formaldehyde solution was then mixed 1:1 with $2 \times$ PBS (1× PBS contains $2.7 \mathrm{mM} \mathrm{KCl}, 1.47 \mathrm{mM} \mathrm{KH} \mathrm{PO}_{4}, 137$ $\mathrm{mM} \mathrm{NaCl}, 8 \mathrm{mM} \mathrm{Na}_{2} \mathrm{HPO}_{4}, \mathrm{pH} 7.4$ ) and Triton X-100 was then added to a final concentration of $0.1 \%$ which provides uniform fixation with reduced cell shrinkage. Fixed cells were washed $2 \times 5$ min with PBS and $1 \times 5$ min with $0.5 \%$ MES (2-N-morpholinoethanesulfonic acid) $\mathrm{pH}$ 5.8. Cell walls were partially digested $30 \mathrm{~min}$ with chromatographically purified lyophilized enzymes from Worthington Biochemical Corporation (Lakewood, NJ, USA). The enzyme mixture was $1 \%$ cellulase (Cat no: LS02601) and 0.5\% pectinase (Cat. no: LS04297) in 0.5\% MES, pH 5.8. After washing with PBS $(3 \times 5 \mathrm{~min})$, cells were settled on poly-L-lysine coated multiwell slides, excess solution was removed and cells were permeabilized 30 min with $0.5 \%$ Triton X-100 in PBS to allow antibody penetration. Following $3 \times 5$ min washes with PBS containing $5 \mathrm{mM} \mathrm{MgSO}_{4}$, cells were incubated $30 \mathrm{~min}$ in 20 units/ml chromatographically purified, ribonuclease- and protease-free DNase I (Worthington Biochem. Corp. cat. no: LS006331) in PBS with $5 \mathrm{mM} \mathrm{MgSO}_{4}$. Fifty times concentrated stock solution of DNase I (1000 units $/ \mathrm{ml}$ ) was prepared by dissolving 2500 units of lyophilized powder in $1.25 \mathrm{ml} \mathrm{50 \%} \mathrm{glycerol} \mathrm{with} 1 \mathrm{mM} \mathrm{CaCl}_{2}$ and kept in freezer in aliquots. DNase I solution was removed with $3 \times 5$ min washes with antibody buffer. Antibody buffer, $\left(\mathrm{PBS}^{+}\right)$contains PBS with 5\% (v/v) fish gelatin (Sigma cat. no: G7765, to prevent nonspecific binding of antibodies) and $0.02 \%$ (w/v) sodium azide (Fluka cat. no: 71290, to inhibit bacterial growth during antibody incubations. For EdU assay sodium azide should not be used before click reaction). Cells were incubated $3 \mathrm{~h}$ at $37^{\circ} \mathrm{C}$ with monoclonal (clone BU-33) mouse anti-bromodeoxyuridine antibody (Sigma cat. no: B8434) diluted 1:200 in $\mathrm{PBS}^{+}$. Following $5 \times 5 \mathrm{~min}$ washes with $\mathrm{PBS}^{+}$, cells were incubated $1 \mathrm{~h}$ at $37^{\circ} \mathrm{C}$ with rabbit anti-mouse Alexa Fluor 488 conjugated antibody (Invitrogen cat no: A11059) diluted 1:300 in $\mathrm{PBS}^{+}$. Cells were then washed $3 \times 5$ min with PBS containing $100 \mathrm{ng} /$ ml DNA staining dye DAPI (4',6-diamidino-2-phenylindole, Invitrogen cat no: D1306) and mounted with Fluoromount-G anti-fade mounting solution (Southern Biotech, cat no: 0100-01).

\section{EdU-based proliferation assay}

Two-days-old monocot and dicot plant suspension cultures were incubated 2 hrs with $10 \mu \mathrm{M}$ EdU (Invitrogen cat no: A10044) in their own culture medium. Arabidopsis cultures (36 h-old) were incubated with various concentrations and durations as shown in Figure 4. Cells were then fixed $15 \mathrm{~min}$ in $4 \%(\mathrm{w} / \mathrm{v})$ formaldehyde solution in phosphate buffered saline (PBS) with $0.1 \%$ Triton X-100. Addition of the detergent Triton X-100 in the fixer prevents cell shrinkage and it also partially permeabilizes the plasma membranes for small detection reagents of EdU assay. Moreover, quick penetration of the fixer allows better preservation of mitotic chromosomes. However, for experiments where preservation of cytoskeleton or cytoplasmic organelles is important, detergent should be omitted from the fixer and plasma membranes should be permeabilized after fixation as in BrdU immunolocalization protocol. Following $3 \times 5 \mathrm{~min}$ PBS washes, 20-30 $\mu$ l packed cell volume of cells were directly incubated $30 \mathrm{~min}$ at room temperature (RT) in EdU detection cocktail (Invitrogen, Click-iT EdU Alexa Fluor $488 \mathrm{HCS}$ assay, cat no: A10027). For 1 sample reaction, following amounts of the kit components are mixed in $144 \mu \mathrm{l}$ distilled water: $1.6 \mu \mathrm{l}$ buffer additive (component $\mathrm{F}$, kept frozen in small aliquots), $14 \mu \mathrm{l}$ reaction buffer (Component D), $6.7 \mu$ l Copper (II) sulfate solution (Component E, $100 \mathrm{mM} \mathrm{CuSO}_{4}$ ) and $0.07 \mu \mathrm{l}$ Alexa Fluor 488 azide (Component B, in $70 \mu$ l DMSO). Many azide-labeled fluorochromes other than Alexa Fluor 488 are available throughout the visible spectrum for multicolor labeling purposes. Click reaction requires $\mathrm{Cu}$ (I) which can be formed using $\mathrm{CuSO}_{4}$ in the presence of a reducing agent such as sodium ascorbate [28]. For experiments where defined buffer components are necessary, we have found that the use of detection cocktail with the following composition resulted in positive EdU labeling on Arabidopsis suspension cultures and on isolated rice and alfalfa nuclei: $4 \mathrm{mM} \mathrm{CuSO}_{4}, 40$ mM sodium ascorbate, $20 \mu \mathrm{M}$ Alexa Fluor 488 azide in PBS (for intact cells) or in nuclei isolation buffer (for nuclei). To prevent oxidation of $\mathrm{Cu}$ (I) to non-catalytic $\mathrm{Cu}$ (II) species, the detection cocktail should be 
prepared freshly. Although the click reaction is not light sensitive, fluorochrome-containing solutions should not be exposed to strong light. After $2 \times 5$ min washes with PBS containing $100 \mathrm{ng} / \mathrm{ml}$ DAPI, an aliquot of cells were mounted in PBS. Glycerol-based (or high osmolarity) mounting mediums caused shrinkage during mounting; therefore PBS mounting is used for all cell lines. For root tip labeling, root tips of germinating rice seedlings (O. sativa L. ssp. japonica 'Nipponbare') were submerged into $20 \mu \mathrm{M}$ (Figure 6) or 0, 2, 20, $100 \mu \mathrm{M}$ EdU (Figure 7) in half strength MS medium. Root tips were then cut in detergent-containing fixer (see above) and fixed for $30 \mathrm{~min}$ at RT. Fixer was washed with PBS $(3 \times$ $10 \mathrm{~min}$ ) and root tips were incubated in EdU detection cocktail (see above) for $30 \mathrm{~min}$ followed by PBS or PBSDAPI washes (see above). Fluoromount-G anti-fade solution was used for mounting of root tips.

\section{Confocal laser scanning and fluorescence stereo microscopy}

Confocal laser scanning microscopy was performed using Olympus Fluoview FV1000 confocal laser scanning microscope (Olympus Life Science Europa GmbH, Hamburg, Germany). Microscope configuration was the following: objective lenses: UPLSAPO 20× (dry, NA:0.75), UPLFLN 40× (oil, NA:1.3) and UPLSAPO 60× (oil, NA:1.35); sampling speed: $4 \mu \mathrm{s} /$ pixel; line averaging: $2 \mathrm{x}$; scanning mode: sequential unidirectional; excitation: $405 \mathrm{~nm}$ (DAPI and cell wall lignin autofluorescence) and $488 \mathrm{~nm}$ (Alexa Fluor 488); laser transmissivity: less than $1 \%$ and $5 \%$ were used for DAPI and Alexa Fluor 488, respectively; main dichroic beamsplitter: DM405/ 488; intermediate dichroic beamsplitter: SDM 490; DAPI and cell wall autofluorescence were detected between 425-475 nm and Alexa Fluor 488 was detected between 500-600 nm and pseudocolored red and green, respectively. Differential interference contrast (DIC) images were captured with $488 \mathrm{~nm}$ laser line. For rice root tip imaging and EdU-signal quantitation, single optical sections of $6 \mu \mathrm{m}$ (optical depth) on the median plane of rice root tips were captured with $20 \times$ objective. Identical laser power and detection settings were used for quantitative analyses. For imaging of all root tips of Figure 7, a higher detector sensitivity setting (as compared to Figure 6) was used due to very short EdU pulse durations. The center of the root with the widest girth was determined by lignin autofluorescence signal of the cell walls. For quantitation of the green EdU signal, meristem regions were manually traced up to $500 \mu \mathrm{m}$ distance starting from the quiescent center. Area measure tool of Olympus Fluoview software (version 1.7.2.2) was used for measurement of area and intensities at the root tips. Total intensity of the green signal (named as "integration" by Olympus Fluoview software) was divided by the measured meristem area to determine average intensity per square microns and plotted using Microsoft Office Excel 2003 software. For nuclear counterstaining and close-up view of chromosomes, DAPI (200 ng/ml) was used on EdU labeled ( 2 hrs) roots and observed by $40 \times$ objective. For fluorescence stereo microscopy, Olympus SZX12 stereo microscope was used. Alexa Fluor 488 images were captured with the "green" filter set: Excitation: 460-490 nm, dichroic beam splitter $505 \mathrm{~nm}$, emission: 510-550 nm. Transmission photos were pseudocolored red and merged with fluorescence images. Composite images were prepared using "import image sequence" and "make montage" functions of ImageJ software (National Institutes of Health, USA, version 1.41).

\section{Nuclei isolation and Flow cytometry}

Three-days-old rice and alfalfa cultures were incubated with $10 \mu \mathrm{M}$ EdU for $8 \mathrm{hrs}$ and $4 \mathrm{hrs}$, respectively. For unfixed nuclei preparations, 4 d-old rice cells were 10 $\mu \mathrm{M}$ EdU-treated for $4 \mathrm{hrs}$. Three-days-old Arabidopsis cultures were incubated either with $0.1 \%$ DMSO (as control) or with $10 \mu \mathrm{M}$ EdU in DMSO for 15' and 30'. EdU-labeled and 0.1\% DMSO-treated control cultures were chopped with a sharp razor blade in nuclei isolation buffer (45 mM $\mathrm{MgCl}_{2}, 20 \mathrm{mM}$ MOPS, $30 \mathrm{mM}$ sodium citrate, $0.1 \%$ Triton $\mathrm{X}-100, \mathrm{pH} \mathrm{7.0)}$ in $6 \mathrm{~cm}$ plastic Petri dishes on ice [17]. Nuclei (in $2 \mathrm{ml}$ buffer) were filtered into $15 \mathrm{ml}$ conical bottom tubes through $20 \mu \mathrm{m}$ sieves and fixed on ice for $30 \mathrm{~min}$ by the addition of $8 \%$ formaldehyde solution (see above) to a final concentration of $1 \%$. Fixed nuclei were washed twice with $2 \mathrm{ml} 0.01 \%$ Triton X-100 containing PBS by centrifugation at $4^{\circ} \mathrm{C}(10 \mathrm{~min}, 400 \mathrm{~g} / 1500 \mathrm{rpm})$ using Heraeus Labofuge 400R (Thermo Fisher Scientific, Rockford, IL, USA) desktop centrifuge with swing-out rotor. Washed nuclei were incubated in $500 \mu \mathrm{l} \mathrm{EdU-detection} \mathrm{cocktail}$ for $30 \mathrm{~min}$ at room temperature. Unfixed nuclei were centrifuged (10') and resuspended in $500 \mu \mathrm{l} \mathrm{EdU-detec-}$ tion cocktail and incubated 30' at room temperature (Water and buffer component D in the cocktail recipe were replaced by nuclei isolation buffer for unfixed nuclei). After one wash (5') with PBS containing $0.01 \%$ Triton X-100 (for fixed nuclei) or with nuclei isolation buffer (for unfixed nuclei), nuclei were counterstained either with $100 \mathrm{ng} / \mathrm{ml}$ DAPI (for microscopy check) or with $5 \mu \mathrm{g} / \mathrm{ml}$ PI (propidium iodide, Invitrogen cat. no: P1304 MP) and analyzed on a FACSCalibur flow cytometer (Becton, Dickinson and Company, NJ, USA) with CellQuest software. Two fluorescence detectors are used with the standard $488 \mathrm{~nm}$ laser. Alexa Fluor 488EdU intensity was detected between 515-545 nm (FL1 channel). For detection of PI intensity (DNA content) 564-606 nm emission range was used at FL2 channel. Side scatter versus forward scatter diagrams were used to locate and gate nuclear populations by particle size. 
Dot-plot diagram of "total PI fluorescence of a particle at FL2 channel" (FL2-A) versus "transit time of a particle at FL2 channel" (FL2-W) was used as secondary gating to exclude particles which are not fluorescent with PI staining. To locate the boundary of EdU-Alexa Fluor 488-labeled nuclei in biparametric plots (EdU threshold value) counts versus FL1-H (Alexa488-EdU channel, log scale) histograms were used. The left (major) peak of this histogram (with low green channel intensity) represents EdU unlabeled G1 and G2 populations while the higher green intensity second peak represents EdUlabeled nuclei. The right border of the leftmost major peak (where the unlabeled G1/G2 counts reach zero value) is selected as the EdU threshold value. EdU threshold values of control samples were determined by corresponding EdU-treated samples. The midpoint PI intensity ( 200 PI intensity units) is selected as the vertical separating line for $2 \mathrm{C}$ DNA and $4 \mathrm{C}$ DNA contents in quadrant analyses.

\section{Acknowledgements}

We would like to thank Katalin Török for technical assistance with plant cell cultures and Zsuzsanna Kószó for technical assistance during experiments involving microscopy. This work was funded by the Hungarian National Research Foundation (OTKA grant no: NK69227)

\section{Author details}

${ }^{1}$ Cellular Imaging Laboratory, Biological Research Center, Hungarian Academy of Sciences, Temesvári krt 62, 6726 Szeged, Hungary. ${ }^{2}$ Institute of Plant Biology, Biological Research Center, Hungarian Academy of Sciences, Temesvári krt 62, 6726 Szeged, Hungary.

\section{Authors' contributions}

EK and FA performed the experimental work. DD and GVH acquired funding, provided plant materials and revised the manuscript. FA designed and coordinated the project. All authors read and approved the final manuscript.

\section{Competing interests}

The authors declare that they have no competing interests.

Received: 5 August 2009

Accepted: 28 January 2010 Published: 28 January 2010

\section{References}

1. Taylor JH, Woods PS, Hughes WL: The organization and duplication of chromosomes as revealed by autoradiographic studies using tritiumlabeled thymidine. Proc Natl Acad Sci USA 1957, 43:122-128.

2. Gratzner HG: Monoclonal antibody to 5-bromo- and 5-iododeoxyuridine: A new reagent for detection of DNA replication. Science 1982 218:474-475.

3. Stroobants C, Sossountzov L, Miginiac E: DNA Synthesis in Excised Tobacco Leaves after Bromodeoxyuridine Incorporation: Immunohistochemical Detection in Semi-thin Spurr Sections. J Histochem Cytochem 1990, 38:641-647.

4. Fowke LC, Cutler AJ: Plant protoplast techniques. Plant Cell Biology. A Practical Approach Oxford, IRL PressHarris N, Oparka KJ 1994, 177-196.

5. Goodbody KC, Lloyd CW: Immunofluorescence techniques for the analysis of the cytoskeleton. Plant Cell Biology. A Practical Approach Oxford, IRL PressHarris N, Oparka KJ 1994, 221-243.

6. Salic A, Mitchison TJ: A chemical method for fast and sensitive detection of DNA synthesis in vivo. Proc Natl Acad Sci USA 2008, 105:2415-2420.

7. Kolb HC, Sharpless KB: The growing impact of click chemistry on drug discovery. Drug Discov Today 2003, 8:1128-1137.
8. Kaiser CL, Kamien AJ, Shah PA, Chapman BJ, Cotanche DA: 5-Ethynyl-2'deoxyuridine labeling detects proliferating cells in the regenerating avian cochlea. Laryngoscope 2009, 119:1770-1775.

9. Warren M, Puskarczyk K, Chapman SC: Chick embryo proliferation studies using EdU labeling. Dev Dyn 2009, 238:944-949.

10. Diermeier-Daucher S, Clarke ST, Hill D, Vollmann-Zwerenz A, Bradford JA, Brockhoff G: Cell type specific applicability of 5-ethynyl-2'-deoxyuridine (EdU) for dynamic proliferation assessment in flow cytometry. Cytometry A 2009, 75:535-546.

11. Limsirichaikul S, Niimi A, Fawcett H, Lehmann A, Yamashita S, Ogi T: A rapid non-radioactive technique for measurement of repair synthesis in primary human fibroblasts by incorporation of ethynyl deoxyuridine (EdU). Nucleic Acids Res 2009, 37:e31.

12. Vanstraelen $M$, Baloban $M$, Da Ines $O$, Cultrone $A$, Lammens $T$, Boudolf $V$, Brown SC, De Veylder L, Mergaert P, Kondorosi E: APC/C-CCS52A complexes control meristem maintenance in the Arabidopsis root. Proc Natl Acad Sci USA 2009, 106:11806-11811.

13. Buck SB, Bradford J, Gee KR, Agnew BJ, Clarke ST, Salic A: Detection of Sphase cell cycle progression using 5-ethynyl-2'-deoxyuridine incorporation with click chemistry, an alternative to using 5-bromo-2'deoxyuridine antibodies. BioTechniques 2008, 44:927-929.

14. Yokochi T, Gilbert DM: Replication labeling with halogenated thymidine analogs. Curr Protoc Cell Biol 2007, 22:22.10

15. Zhang $L$, Chen $X$, Xue $P$, Sun HH, Williams ID, Sharpless KB, Fokin W, Jia G Ruthenium-catalyzed cycloaddition of alkynes and organic azides. J Am Chem Soc 2005, 127:15998-15999.

16. Popper ZA: Evolution and diversity of green plant cell walls. Curr Opin Plant Biol 2008, 11:286-292.

17. Galbraith DW, Harkins KR, Maddox JM, Ayres NM, Sharma DP, Firoozabady E: Rapid flow cytometric analysis of the cell cycle in intact plant tissues. Science 1983, 220:1049-1051.

18. Paschalidis KA, Roubelakis-Angelakis KA: Sites and Regulation of Polyamine Catabolism in the Tobacco Plant. Correlations with Cell Division/ Expansion, Cell Cycle Progression, and Vascular Development. Plant Physiol 2005, 138:2174-2184.

19. Zhang C, Gong FC, Lambert GM, Galbraith DW: Cell type-specific characterization of nuclear DNA contents within complex tissues and organs. Plant Methods 2005, 1:7.

20. Lucretti S, Nardi L, Nisini PT, Moretti F, Gualberti G, Dolezel J: Bivariate flow cytometry DNA/BrdUrd analysis of plant cell cycle. Methods Cell Sci 1999, 21:155-166.

21. Sasaki K, Adachi S, Yamamoto T, Murakami T, Tanaka K, Takahashi M: Effects of denaturation with $\mathrm{HCl}$ on the immunological staining of bromodeoxyuridine incorporated into DNA. Cytometry 1988, 9:93-96.

22. Harris N: Immunocytochemistry for light and electron microscopy. Plant Cell Biology. A Practical Approach Oxford, IRL PressHarris N, Oparka KJ 1994 157-176.

23. Chapman S, Oparka KJ, Roberts AG: New tools for in vivo fluorescence tagging. Curr Opin Plant Biol 2005, 8:565-573.

24. Tsukaya $\mathrm{H}$ : Determination of the unequal fate of cotyledons of a one-leaf plant, Monophyllaea. Development 1997, 124:1275-1280.

25. de Castro RD, van Lammeren AA, Groot SP, Bino RJ, Hilhorst HW: Cell division and subsequent radicle protrusion in tomato seeds are inhibited by osmotic stress but DNA synthesis and formation of microtubular cytoskeleton are not. Plant Physiol 2000, 122:327-335.

26. Breuil-Broyer S, Morel P, de Almeida-Engler J, Coustham V, Negrutiu I, Trehin C: High-resolution boundary analysis during Arabidopsis thaliana flower development. Plant J 2004, 38:182-192.

27. Kinoshita I, Sanbe A, Yokomura El: Difference in light-induced increase in ploidy level and cell size between adaxial and abaxial epidermal pavement cells of Phaseolus vulgaris primary leaves. J Exp Bot 2008, 59:1419-1430.

28. Rodionov VO, Fokin W, Finn MG: Mechanism of the Ligand-Free Cu (I)Catalyzed Azide-Alkyne Cycloaddition Reaction. Angew Chem Int Ed 2005, 44:2210-2215.

29. Murashige T, Skoog F: A revised medium for rapid growth and bioassays with tobacco cultures. Physiol Plant 1962, 15:473-497.

30. Mórocz S, Donn G, Németh J, Dudits D: An improved system to obtain fertile regenerants via maize protoplasts isolated from a highly embryogenic suspension culture. Theor Appl Genet 1990, 80:721-726. 
31. Pauk J, Nerlich A, Pawelczyk H, Schledzewski K, Matuz J, Simon IK, Mendel RR: Transgenic fertile rice plants obtained through biolistic transformation using reporter genes and TR promoter. Rice Genetics III Manila, PhilippinesKush GS 1996, 719-722.

32. Jenes B, Pauk J: Plant regeneration from protoplast derived calli in rice (Oryza sativa L.) using Dicamba. Plant Science 1989, 63:187-198.

doi:10.1186/1746-4811-6-5

Cite this article as: Kotogány et al: A rapid and robust assay for detection of S-phase cell cycle progression in plant cells and tissues by using ethynyl deoxyuridine. Plant Methods 2010 6:5

Submit your next manuscript to BioMed Central and take full advantage of:

- Convenient online submission

- Thorough peer review

- No space constraints or color figure charges

- Immediate publication on acceptance

- Inclusion in PubMed, CAS, Scopus and Google Scholar

- Research which is freely available for redistribution

Submit your manuscript at www.biomedcentral.com/submit
C Biomed Central 